\title{
ATHK1 acts downstream of hydrogen peroxide to mediate ABA signaling through regulation of calcium channel activity in Arabidopsis guard cells
}

\author{
LÜ Dong, WANG Wei \& MIAO Chen ${ }^{*}$ \\ State Key Laboratory of Cotton Biology, Key Laboratory of Plant Stress Biology, Henan University, Kaifeng 475004, China
}

Received January 25, 2012; accepted April 9, 2012; published online October 29, 2012

Plants gradually develop their ability to tolerate environmental water deficit as part of the evolutionary process. Abscisic acid (ABA) plays a critical role during drought and osmotic stress. Several histidine protein kinases are regarded as osmotic sensors or regulators in the adaptive response of plants to water deficit. In this study, we report that ATHK1, which was previously shown to function as an osmotic regulator, is involved in ABA-induced stomatal signaling in Arabidopsis. Mutants null for ATHK1 expression were unable to transmit normal ABA responses in guard cells, including inducing stomatal closure, producing hydrogen peroxide and activating calcium influx. Moreover, patch clamp and confocal analysis demonstrated that ATHK1 may function downstream of hydrogen peroxide in ABA-induced stomatal closure, by regulating calcium channel activity and calcium oscillation in Arabidopsis guard cells.

ATHK1, $\mathrm{H}_{2} \mathrm{O}_{2}, \mathrm{ABA}$, calcium channels, guard cell

Citation: Lü D, Wang W, Miao C. ATHK1 acts downstream of hydrogen peroxide to mediate ABA signaling through regulation of calcium channel activity in Arabidopsis guard cells. Chin Sci Bull, 2013, 58: 336-343, doi: 10.1007/s11434-012-5207-y

Plant stomata are bordered by specialized cells known as guard cells, which control the exchange of carbon dioxide and oxygen, thereby regulating respiration and transpiration. When faced with environmental variation, plants maintain their growth through a series of complicated systems that involves crucial processes, one of which includes the regulation of stomatal movement. Various signal transduction pathways function in guard cells to regulate stomatal opening or closure, hence these cells are an ideal model for studying mechanisms controlling these processes [1].

The plant hormone, abscisic acid (ABA), induces stomatal closure in higher plants as an important response in resistance to drought [2]. In the process of ABA-induced stomatal closure, several signaling components are involved. These include small molecules such as hydrogen peroxide $\left(\mathrm{H}_{2} \mathrm{O}_{2}\right)$, and ion channels such as calcium channels and potassium channels [3-7].

Several Arabidopsis thaliana histidine protein kinases (ATHKs) have been shown to act as regulators participating

*Corresponding author (email: miaochen928@ henu.edu.cn) in important mechanisms of stress resistance. Indeed, numerous studies have reported that Arabidopsis thaliana histidine kinase 1 (ATHK1) specifically responds to osmotic stress [8-11]. The ATHK1 gene was previously cloned by Urao et al. [8,12], and encodes a heterozygous histidine protein kinase. The protein consists of two trans-membrane domains, which are sensitive to environmental osmotic changes, similar to those present in yeast histidine kinases. Researchers have shown that ATHKI mRNA is highly expressed in root tissue, compared with other tissues, and rapidly accumulates in response to changes in soil salinity or osmotic variation, thus making it a highly efficient sensor to environmental fluctuations $[8,12,13]$. Recent research revealed that ATHK1 plays a unique role in the regulation of processes controlling desiccation, which occur during seed formation. Mechanistically, this may involve regulation through ABA mediated pathways, since overexpression of ATHK1 led to increased transcription of ABA biosynthetic genes [14]. However, while ATHK1 has been implicated in ABA signaling, the exact role and position of ATHK1 in this pathway are unclear. In previous studies, we demon- 
strated a role for secondary signaling molecules, such as hydrogen peroxide $\left(\mathrm{H}_{2} \mathrm{O}_{2}\right)$ and ion channels, such as the calcium $\left(\mathrm{Ca}^{2+}\right)$ channel in ABA-induced stomatal movement $[6,7,15]$. Because ATHK1 also interferes with stomatal movement, we investigated the potential for crosstalk between ATHK1 and ABA- $\mathrm{H}_{2} \mathrm{O}_{2}$ signaling and the mechanisms underlying the interaction of these pathways.

\section{Materials and methods}

\subsection{Chemicals}

ABA, $\mathrm{H}_{2} \mathrm{O}_{2}$, dichlorofluorescin diacetate $\left(\mathrm{H}_{2} \mathrm{DCF}-\mathrm{DA}\right)$, acetyloxymethyl 2-[5-[2-[5-[3-[4-[2-(acetyloxymethoxy)2-oxoethyl]piperazin-1-yl]-3-oxopropyl]-2-[bis[2-(acetylox ymethoxy)-2-oxoethyl]amino]phenoxy]ethoxy]-6-[bis[2(acetyloxymethoxy)-2-oxoethyl]amino]-1-benzofuran-2yl]-1,3-oxazole-5-carboxylate (FURA3-AM), nicotinamide adenine dinucleotide phosphate (NADPH) diphenyleneiodonium Chloride (DPI), were purchased from Sigma Aldrich (USA). Analytical grade 2- $N$-morpholino ethanesulfonic acid (MES) and dimethyl sulfoxide (DMSO)(Sigma Aldrich) were used. Prior to use, all chemicals were dissolved in solvents in accordance with their qualities and manufacturer's instructions.

\subsection{Epidermal strip bioanalysis and measurement of water loss}

Thin strips were peeled from Arabidopsis leaves using medical tweezers and $\sim 0.5 \mathrm{~cm}^{2}$ sections were incubated in MES-KCl buffer ( $\mathrm{KCl} 50 \mathrm{mmol} \mathrm{L}^{-1}$, MES $10 \mathrm{mmol} \mathrm{L}^{-1}$ ) for $1 \mathrm{~h}$. Prior to treatment with reagents, stomata pores were opened by exposure to $0.3 \mathrm{mmol} \mathrm{m}^{-2} \mathrm{~s}^{-1}$ light density for $2 \mathrm{~h}$ and the stomatal aperture was recorded using an optical measure microscope (Nikon, magnification 400×). The epidermal strips were then incubated with various reagents (ABA, $\mathrm{H}_{2} \mathrm{O}_{2}$ or DPI) dissolved in MES-KCl buffer for $1 \mathrm{~h}$ and the stomatal aperture was measured. For measurement of water loss, rosette leaves of wild-type and ATHK1deficient mutant strains were detached from 4-week-old seedlings, placed in weighing dishes, and incubated on the laboratory bench at $20^{\circ} \mathrm{C}$. Weight was monitored at the indicated times (for $3 \mathrm{~d}$ continuously) and water loss expressed as the percentage of initial fresh weight at day one.

\subsection{Patch clamp for calcium channel}

Wild-type and ATHK1-deficient strains of Arabidopsis thaliana were grown in a controlled environment chamber with a $16 \mathrm{~h} / 8 \mathrm{~h}$ light/dark cycle. Guard cell protoplasts were isolated from 4- to 6-week plants as previously described [6]. Pipettes were pulled with a vertical puller (Narishige, Japan) modified for two-stage pulls. The whole cell voltage-clamp on Arabidopsis guard cells was recorded using an EPC-9 amplifier (Heka Instrument, Germany) as previously described [6]. Data were analyzed using PULSEFIT 8.7 software. Standard solutions for $\mathrm{Ca}^{2+}$ measurements were used, including $10 \mathrm{mmol} \mathrm{L} \mathrm{BaCl}_{2}, 0.1 \mathrm{mmol} \mathrm{L} \mathrm{mTT}^{-1} \mathrm{DT}, 10 \mathrm{mmol}$ $\mathrm{L}^{-1}$ MES-Tris (pH 5.6) in the bath, and $100 \mathrm{mmol} \mathrm{L}^{-1} \mathrm{BaCl}_{2}$ 0.1 mmol L ${ }^{-1}$ DTT, 4 mmol L ${ }^{-1}$ EGTA, $10 \mathrm{mmol} \mathrm{L}^{-1}$ HEPES-Tris ( $\mathrm{pH} 7.1$ ) in the pipette. ABA, $\mathrm{H}_{2} \mathrm{O}_{2}$ and DPI were freshly added to bath solutions at the indicated concentrations. For ABA-activated $\mathrm{Ca}^{2+}$ current measurements, $1 \mathrm{mmol} \mathrm{L}{ }^{-1} \mathrm{NADPH}$ was added to the standard pipette solution. Osmolalities of pipette and bath solutions were ad-

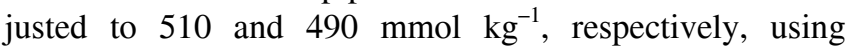
$D$-sorbitol (Sangon, China).

\subsection{Laser scanning confocal microscopy}

$\mathrm{H}_{2}$ DCFDA (50 mmol/L) and FURA3-AM (5 mmol/L) solutions were prepared in DMSO. These were directly added to loading buffers at a final concentration of $10 \mu \mathrm{mol} \mathrm{L}^{-1}$ for FURA3-AM or $50 \mu \mathrm{mol} \mathrm{L}^{-1}$ for $\mathrm{H}_{2}$ DCFDA. During the loading process, the sample temperature was maintained at $25^{\circ} \mathrm{C}$. After loading (60 min for FURA3-AM, $30 \mathrm{~min}$ for $\mathrm{H}_{2}$ DCFDA), epidermal strips were transferred into fresh loading buffer to remove excess probes. The excitation and absorption wave-lengths used were 485 and 515-530 nm, respectively.

\section{Results}

ATHK1 mutants (athk1-4 and CS) were derived from wild-type plants (WS and Col-0), by insertion of a fragment into the promoter region. We examined the T-DNA mutant, derived from SALK_000976 (insertion 88 bp fragment: 5'-CTTCTCTGTGGATGAAGCAGAGNNNTTAGTCNNTCCNANTTTTGAAGTTTTCCTTCNTGGGNTTCTGATTTGGCTACTANAGTTTTGA-3') and CS849900 (insertion 309 bp fragment: 5'-ATTTTCCATAAGCAAAAAGATTTCATCTTTCTATTTATTCTCTTTATAAATTGGAACCTACTTTGCTTCTTCTTCAGTCTCCACACTTATCTTATTTTTGCCAGTCTTCTCTGTGGATGAAGCAGAGTTTTTAGTCAATCAAAAGTTTGAAGCTTTCCTTCATGGGTTTCTGATTTGTCTACTAAAGTTTTGATTTTTTTAATTTGGGTATTAATAAAAAAAGTGGGGAGTGACACAAGAAGCAGTATAGTCATTAGTTTTCCACTAAGCAAAGCACCCAAAAGTTCACCAACACAANACTCGAGAAGA-3'), and observed several differences between mutant and wild-type plants.

\subsection{Loss of ATHK1 is associated with differences in water loss}

To investigate the role of ATHK1 in stomata regulation and transpiration, we used infrared cameras to examine the temperature of leaves from both wild-type and ATHK1 mutant 
plants. ATHK1 mutants displayed a relatively lower average temperature compared with their wild-type counterparts under conditions of normal humidity (data not shown).

Analysis of water loss also revealed that ATHK1 mutants lost water at a faster rate than wild-type plants. Both ATHK1 mutants lost $10 \%$ more water than wild-type plants after 60 min (Figure 1(d)). These results were not explained by differences in stomata numbers, as we observed no distinct differences in the number of stomata per square centimeter between wild-type and mutant plants (Figure 1(c)).

Taken together, these data indicate that ATHK1 inhibits the loss of water vapor through stomata. We next asked whether ATHK1-regulated stomatal movement is controlled by $\mathrm{ABA}$.

\subsection{ATHK1 is involved in $\mathrm{ABA}$ and $\mathrm{H}_{2} \mathrm{O}_{2}$-induced stomatal closure in Arabidopsis thaliana}

To investigate the involvement of ATHK1 in stomatal
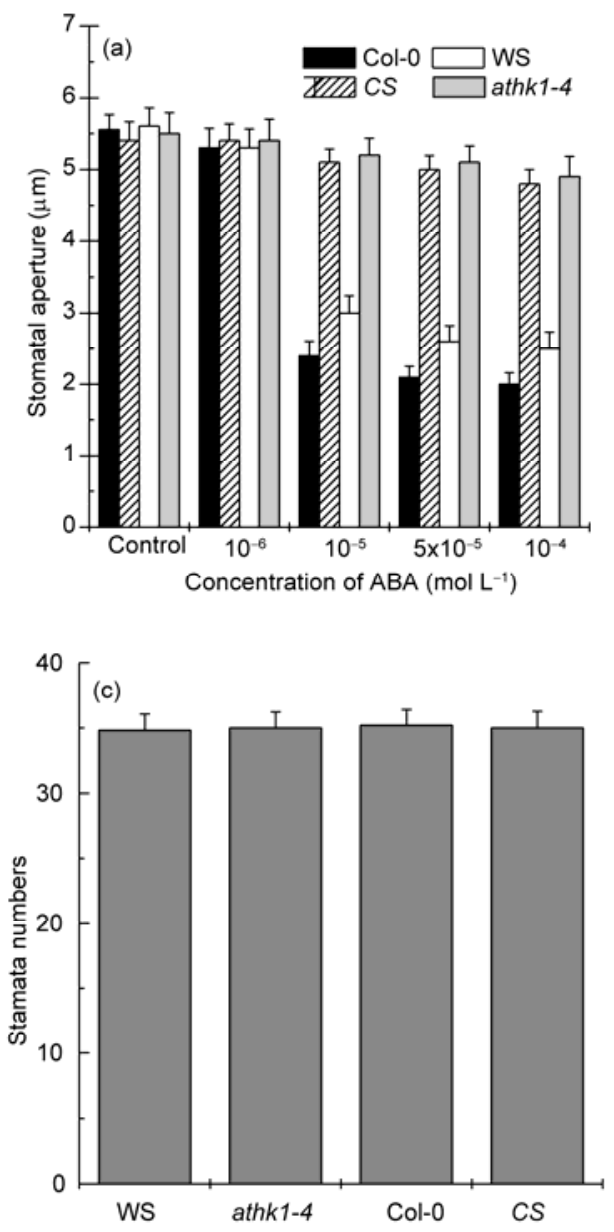

closure induced by $\mathrm{ABA}$, we assayed the response of wild-type and ATHK1 mutant plants using the epidermal stomatal bioassay, which is typically used to test the function of ABA (Figure 1(a)). We also tested the effect of $\mathrm{H}_{2} \mathrm{O}_{2}$ on stomata in these plant lines, owing to its secondary signaling role in ABA-induced stomatal closure (Figure 1(b)).

As shown in Figure 1(a) and (b), treatment of plants with a range of concentrations of ABA $\left(0-100 \mu \mathrm{mol} \mathrm{L}{ }^{-1}\right)$ and $\mathrm{H}_{2} \mathrm{O}_{2}$ $\left(0-500 \mu \mathrm{mol} \mathrm{L}{ }^{-1}\right)$, revealed that ATHK1 mutants were less sensitive to $\mathrm{ABA}$ or $\mathrm{H}_{2} \mathrm{O}_{2}$ than their wild-type counterparts. These differences were most striking for ABA at $10 \mu \mathrm{mol} \mathrm{L} \mathrm{L}^{-1}$ and for $\mathrm{H}_{2} \mathrm{O}_{2}$ at $100 \mu \mathrm{mol} \mathrm{L} \mathrm{L}^{-1}$. These results suggest that ATHK1 plays a role in ABA- $\mathrm{H}_{2} \mathrm{O}_{2}$ signaling in guard cells. We next asked how ATHK1 performs this function.

\subsection{ATHK1 does not inhibit ABA-induced $\mathrm{H}_{2} \mathrm{O}_{2}$ pro- duction in guard cells}

ABA induces $\mathrm{H}_{2} \mathrm{O}_{2}$ production as a classic response to
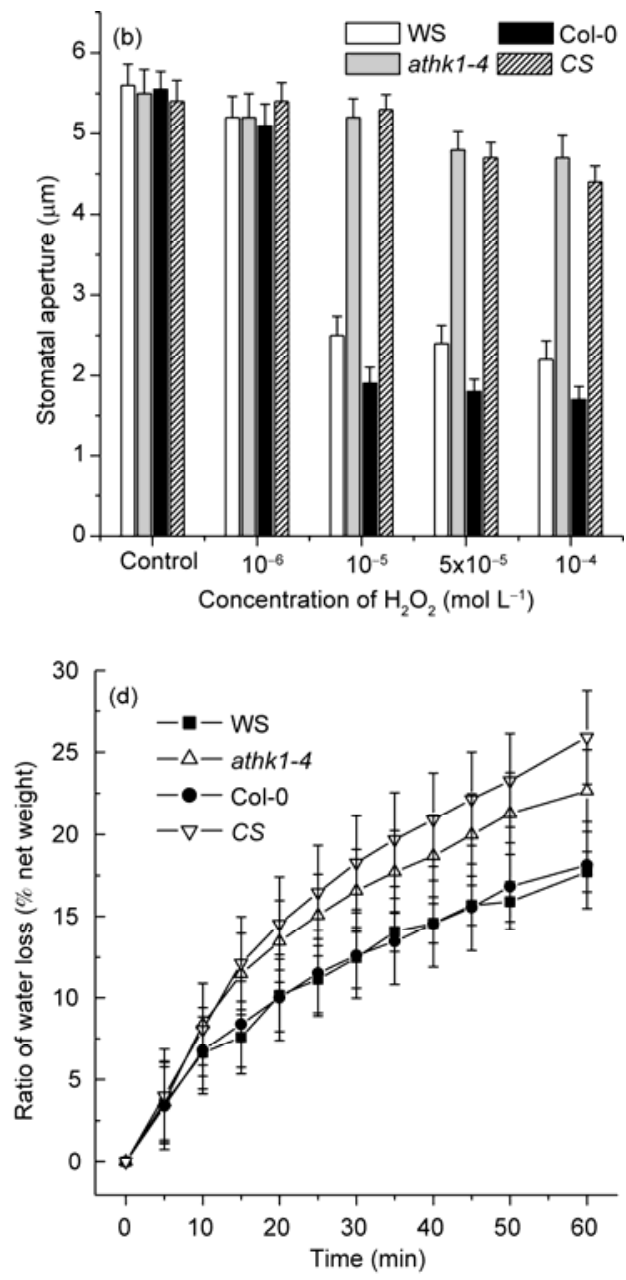

Figure 1 ATHK1 plays a role in ABA and $\mathrm{H}_{2} \mathrm{O}_{2}$-induced stomatal closure. (a) Stomatal aperture of wild-type plants (WS and Col-0) or ATHK1 mutants (athk1-4 and CS) at given ABA concentrations $\left(n \geqslant 250\right.$ ). (b) Stomatal aperture of wild-type or ATHK1 mutant plants at given $\mathrm{H}_{2} \mathrm{O}_{2}$ concentrations $(n \geqslant$ 250). (c) Determination of stomatal cell patterning between wild-type and ATHK1 mutant plants. (d) Water loss ratio of detached plants of ATHK1 mutants $(n=5)$ was higher than that of wild-type plants $(n=5)$. Data for (c) and (d) represent the average of 5 independent experiments $\pm \operatorname{SEM}(P \leqslant 0.05)$. 
drought in guard cells [2]. To assess the role of ATHK1 in $\mathrm{H}_{2} \mathrm{O}_{2}$ production, we performed $\mathrm{H}_{2}$ DCF fluorescence assays to measure $\mathrm{H}_{2} \mathrm{O}_{2}$ levels in both wild-type and mutant plants. Laser scanning confocal microscopy (LSCM) analysis of guard cells revealed that fluorescence levels were 10-fold higher in ABA treated cells compared with controls, in both wild-type and $A T H K 1$ mutant cells. The NADPHase inhibitor, DPI, was capable of inhibiting the production of $\mathrm{H}_{2} \mathrm{O}_{2}$ in both systems (Figure 2). These results suggest that ATHK1 does not affect $\mathrm{H}_{2} \mathrm{O}_{2}$ production in response to ABA treatment.

\subsection{ATHK1 regulates $\mathrm{ABA}$ and $\mathrm{H}_{2} \mathrm{O}_{2}$-induced $\mathrm{Ca}^{2+}$ channel activity in guard cells}

Previous studies by Pei et al. [4] revealed that $\mathrm{H}_{2} \mathrm{O}_{2}$ controls ABA function via the regulation of $\mathrm{Ca}^{2+}$ channels in guard cells. Based on this, we hypothesized that there may be differences in $\mathrm{ABA}$ or $\mathrm{H}_{2} \mathrm{O}_{2}$-regulated $\mathrm{Ca}^{2+}$ channel signaling between wild-type and ATHK1 mutant plants.

To test this, we performed a whole-cell ramp patch clamp. In wild-type plants, treatment with ABA significantly induced $\mathrm{Ca}^{2+}$ channel activity for inward $\mathrm{Ca}^{2+}$ currents compared with control, which rose from $<70 \mathrm{pA}$ at time zero, to $350 \mathrm{pA}$ at $10 \mathrm{~min}$. Treatment with DPI led to a decrease in activity to 170 pA (Figure 3(a) and (c)). In contrast, treatment of mutants with ABA had minimal effect on $\mathrm{Ca}^{2+}$ channel activity compared with controls, with changes no greater than $100 \mathrm{pA}$ observed (Figure 3(b) and (c)).

Similarly, treatment of wild-type guard cells with $\mathrm{H}_{2} \mathrm{O}_{2}$ led to an increase in the $\mathrm{Ca}^{2+}$ current to approximately 350 pA (Figure 3(a) and (d)), whereas in ATHK1 mutant cells, this current was not significantly changed compared with controls, varying less than 100 pA (Figure 3(b) and (d)).

\subsection{ATHK1 affects $\mathrm{ABA}$ and $\mathrm{H}_{2} \mathrm{O}_{2}$-induced $\mathrm{Ca}^{2+}$ con- centration variation in guard cells}

Previous research has shown that $\mathrm{ABA}$ and $\mathrm{H}_{2} \mathrm{O}_{2}$ are capable of inducing changes in the $\mathrm{Ca}^{2+}$ concentration of guard cells, preceding stomatal closure $[4,7] . \mathrm{Ca}^{2+}$ channel signaling on the guard cell membrane is regarded as the main source for elevation of inner-cell $\mathrm{Ca}^{2+}$ concentrations [1]. ABA and $\mathrm{H}_{2} \mathrm{O}_{2}$ induce stomatal closure by affecting the cytoplasmic concentration and dynamic variation of $\mathrm{Ca}^{2+}$. ATHKl deficiency may inhibit $\mathrm{Ca}^{2+}$ channel signaling and the cytoplasmic concentration of $\mathrm{Ca}^{2+}$, thus rendering guard cells insensitive to $\mathrm{ABA}$ and $\mathrm{H}_{2} \mathrm{O}_{2}$. To test this hypothesis, we assessed FURA3-AM fluorescence, which is an indicator of intracellular $\mathrm{Ca}^{2+}$ levels, in wild-type and ATHK1 mutant plants using confocal microscopy.

As shown in Figure 4, treatment with ABA led to an increase in FURA3-AM fluorescence in wild-type WS guard cells, but did not significantly affect fluorescence in ATHK1 mutants. Indeed, ABA-induced fluorescence was 3 -fold higher in wild-type compared with mutant plants. Treatment with both ABA and DPI inhibited FURA3-AM fluorescence induced by ABA in wild-type plants, without affecting fluorescence in mutant plants (Figure 4).
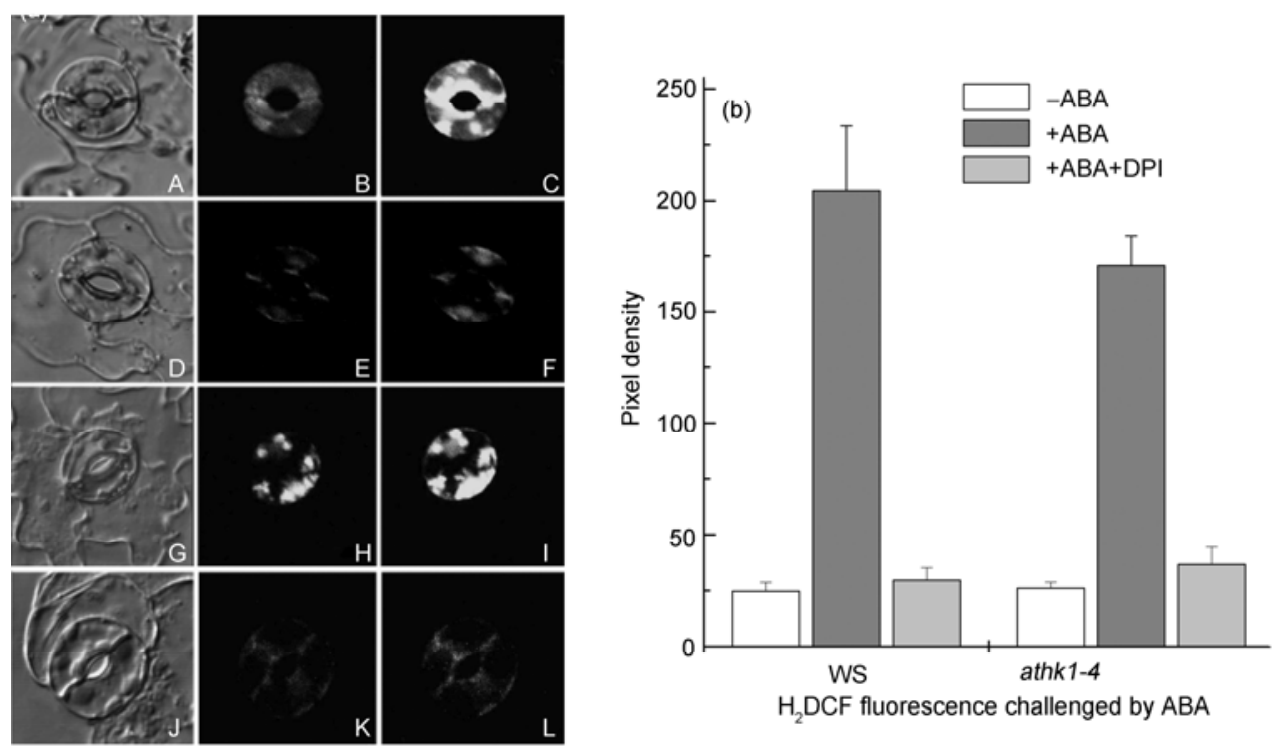

Figure $2 \mathrm{H}_{2}$ DCF fluorescence showing the production of $\mathrm{H}_{2} \mathrm{O}_{2}$ before (B, E, H, K) and after abscisic acid (ABA) treatment (C, F, I, L) in wild-type WS and ATHK1 mutant guard cells. (a) Laser scanning confocal microscopy (LSCM) revealed variations in $\mathrm{H}_{2} \mathrm{DCF}$ fluorescence. A-C, WS+ABA; D-F, WS+ABA+DPI; G-I, ATHK1+ABA; J-L, ATHK1+ABA+DPI. (b) Changes in $\mathrm{H}_{2} \mathrm{DCF}$ fluorescence pixel density in both wild-type and ATHK1 mutant plants after treatment with ABA and ABA + DPI $(n=10)$. Data represent the mean fluorescence intensity for 10 independent guard cells \pm SEM $(P \leqslant 0.05)$. The concentration of reagents are as follows: ABA, $10 \mu \mathrm{mol} \mathrm{L}{ }^{-1}$; DPI, $50 \mu \mathrm{mol} \mathrm{L}^{-1}$. 

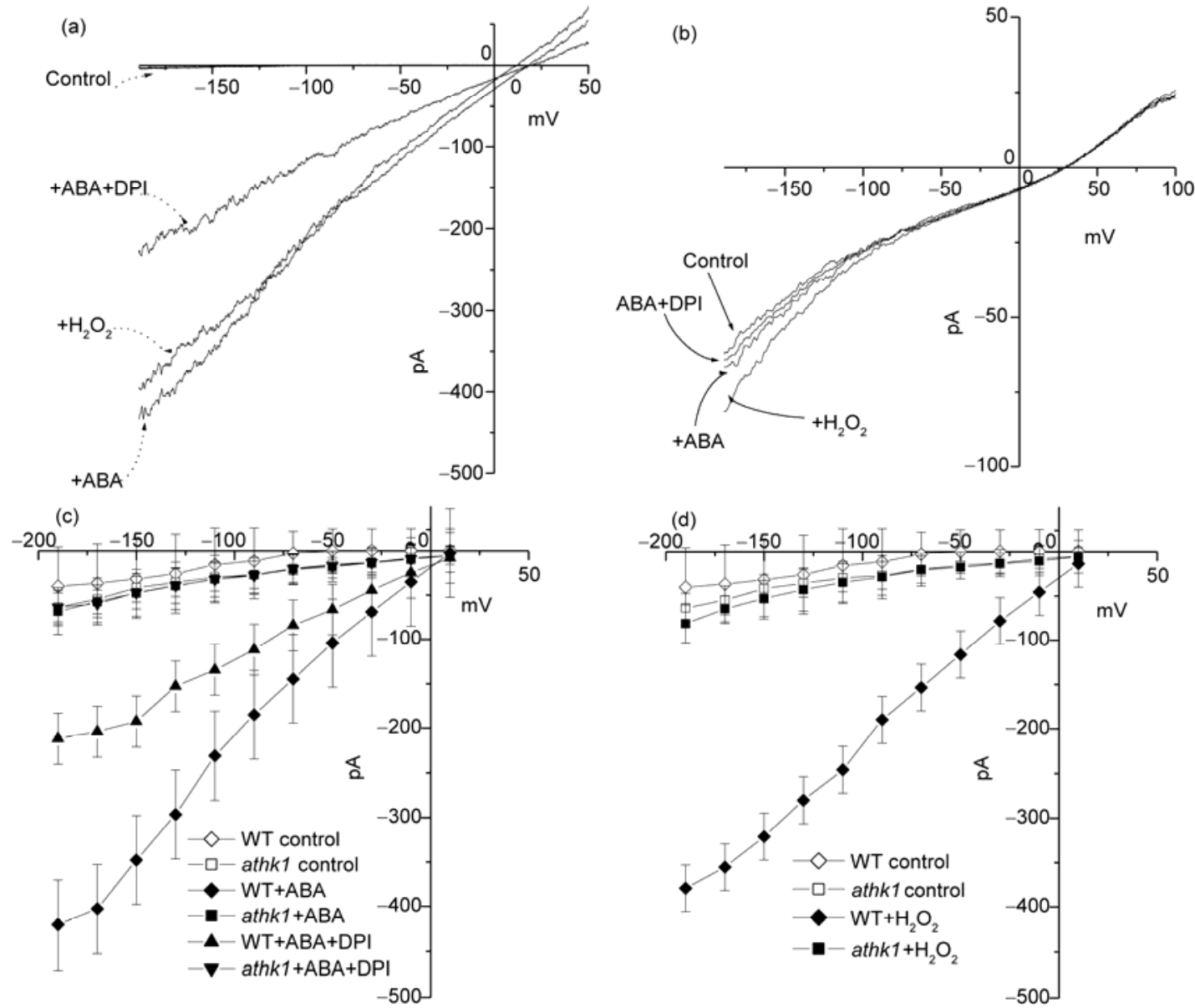

Figure $3 \mathrm{Ca}^{2+}$ channel activity on guard cell membranes is affected by $\mathrm{ABA}$ and $\mathrm{H}_{2} \mathrm{O}_{2}$ in wild-type WS and $A T H K 1$ mutant plants. (a) Ca ${ }^{2+}$ channel activity in WS membrane before and after ABA (and DPI) or $\mathrm{H}_{2} \mathrm{O}_{2}$ treatment. (b) $\mathrm{Ca}^{2+}$ channel activity in ATHK1 mutants before and after ABA (and DPI) or $\mathrm{H}_{2} \mathrm{O}_{2}$ treatment. (c) Statistical analyses of $\mathrm{Ca}^{2+}$ channel currents following ABA exposure in wild-type and ATHK1 mutant plants ( $n=10$ ). (d) Statistical analyses of $\mathrm{Ca}^{2+}$ channel current following $\mathrm{H}_{2} \mathrm{O}_{2}$ treatment in wild-type and ATHK1 mutant plants $(n=10)$. The final concentration of reagents was: ABA, 10 $\mu \mathrm{mol} \mathrm{L}{ }^{-1} ; \mathrm{H}_{2} \mathrm{O}_{2}, 100 \mu \mathrm{mol} \mathrm{L}^{-1}$; DPI, $50 \mu \mathrm{mol} \mathrm{L}^{-1}$.

\subsection{ATHK1 regulates the rhythm of $\mathrm{Ca}^{2+}$ concentra- tion fluctuation}

Oscillation of $\mathrm{Ca}^{2+}$ levels is characteristic of many biological reactions. To test the idea that ATHK1 may affect $\mathrm{Ca}^{2+}$ oscillation in guard cells, we performed a time course experiment to assess calcium levels in wild-type and mutant cells. Our results revealed a fluctuating pattern of $\mathrm{Ca}^{2+}$ concentration in wild-type guard cells upon exposure to ABA (Figure $5(\mathrm{a})$ ). In contrast, no such fluctuations were observed in ATHK1 mutant guard cells (Figure 5(b)). We also observed that the oscillation in $\mathrm{Ca}^{2+}$ concentration only occurred at higher concentrations of $\mathrm{Ca}^{2+}$ (Figure 5(c)).

\section{Discussion}

In this study, we provide evidence that ATHK1 plays a role in ABA signal transduction and control of $\mathrm{H}_{2} \mathrm{O}_{2}$-induced calcium channel activity in guard cells, mediating stomatal closure. ATHK1 mutants displayed less sensitivity to ABA under normal conditions, in contrast with wild-type plants. Compared with wild-type plants, ATHK1 mutants displayed impaired ABA-induced stomatal closure and more rapid water loss in response to water deficit stress (Figure 1). Previous studies have demonstrated the effect of $\mathrm{H}_{2} \mathrm{O}_{2}$ on stomatal closure $[4,6]$. In this study, we found that $\mathrm{H}_{2} \mathrm{O}_{2}$ production induced by ABA was not decreased in ATHK1 mutant guard cells, suggesting that the loss-of-function mutation in ATHK1 does not impair $\mathrm{H}_{2} \mathrm{O}_{2}$ homeostasis in guard cells (Figure 2). It is possible that ATHK1 acts downstream of $\mathrm{H}_{2} \mathrm{O}_{2}$, modulating stomatal closure in guard cells. ATHK1 is not only critical for mediating the effects of $\mathrm{H}_{2} \mathrm{O}_{2}$, but is an essential element of the ABA signaling pathway that mediates stomatal closure. The finding that ATHK1 is involved in ABA signaling in guard cells raises the questions of how $\mathrm{ABA}$ and $\mathrm{H}_{2} \mathrm{O}_{2}$ signals are transduced, and the identities of the components of the cascade involved in this process.

Previous studies have shown that ABA-induced $\mathrm{H}_{2} \mathrm{O}_{2}$ 

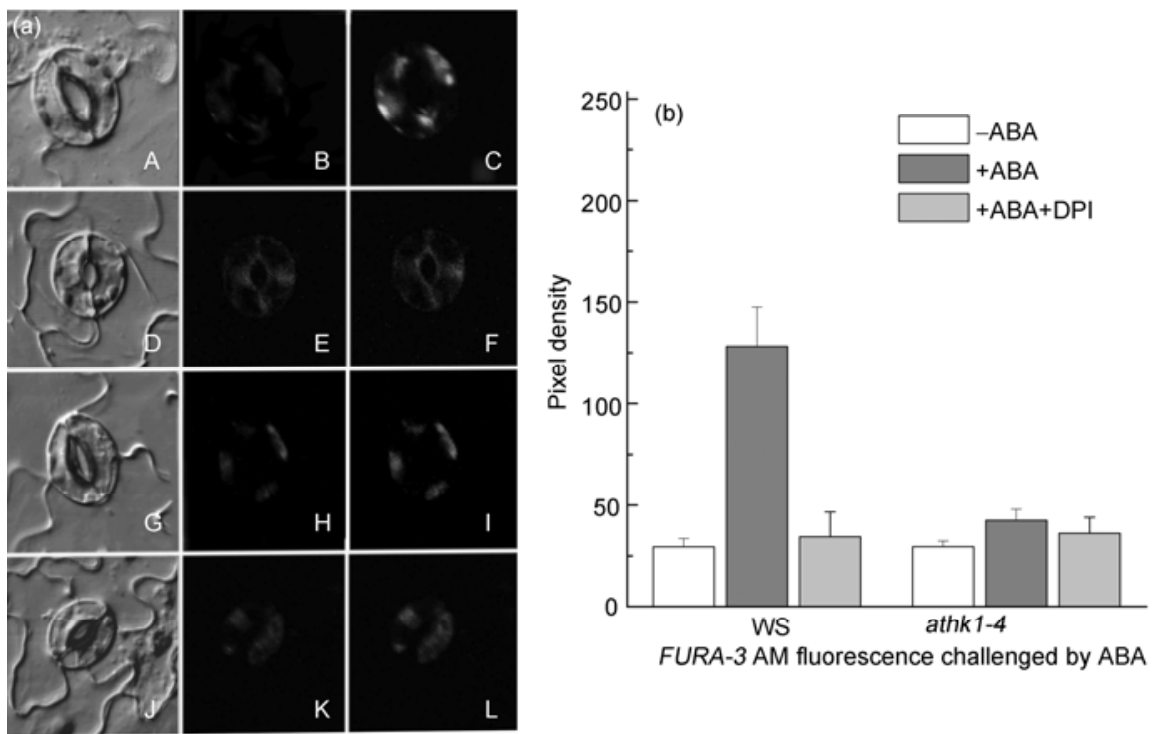

Figure 4 FURA3-AM fluorescence showing changes in intracellular guard cell $\mathrm{Ca}^{2+}$ concentration before $(\mathrm{B}, \mathrm{E}, \mathrm{H}$, and $\mathrm{K})$ and after $\mathrm{ABA}$ treatment $(\mathrm{C}, \mathrm{F}, \mathrm{I}$, L) in wild-type WS and ATHK1 mutant cells. (a) LSCM images display the variation of FURA3-AM fluorescence following treatment with ABA and ABA + DPI. A-C, WS+ABA; D-F, WS+ABA+DPI; G-I, ATHK1+ABA; J-L, ATHK1+ABA+DPI. (b) Changes in FURA3-AM fluorescence pixel density in both wild-type and ATHK1 mutant plants after treatment with ABA and ABA + DPI. Data points are expressed as the mean \pm SEM $(n=10, P \leqslant 0.05)$. The final concentration of reagents was: $\mathrm{ABA}, 10 \mu \mathrm{mol} \mathrm{L}{ }^{-1}$; DPI, $50 \mu \mathrm{mol} \mathrm{L}^{-1}$.

generation in the guard cells of Vicia and Arabidopsis leads to the activation of plasma membrane $\mathrm{Ca}^{2+}$ channels, thus permitting $\mathrm{Ca}^{2+}$ influx in guard cells $[4,5,15]$. The link between ABA-induced $\mathrm{H}_{2} \mathrm{O}_{2}$ production and ATHK1 isfurther strengthened by our observations. In this study, we found that ATHK1 functions downstream of $\mathrm{ABA}$ and $\mathrm{H}_{2} \mathrm{O}_{2}$, but upstream of $\mathrm{Ca}^{2+}$ channel signaling. Mutations in ATHK1 impaired $\mathrm{ABA}$-induced stomatal closure and the activation of $\mathrm{Ca}^{2+}$-permeable channels in the plasma membrane. Indeed, calcium currents were not affected by ABA and $\mathrm{H}_{2} \mathrm{O}_{2}$ in the ATHK1 mutant plants compared with their wild-type counterparts (Figure 3 ). One possibility for this observation is that loss of function of $A T H K 1$ affects downstream events mediated by $\mathrm{H}_{2} \mathrm{O}_{2}$ and $\mathrm{ABA}$ signaling, thereby blocking the activation of $\mathrm{Ca}^{2+}$ channels within guard cells and disrupting stomatal closure. The intimate connection between ATHK1 and $\mathrm{Ca}^{2+}$ channel activity implies that ATHK1 may play an important role in sensing and monitoring intracellular $\mathrm{Ca}^{2+}$ levels in guard cells.

Previous research in our laboratory showed that ABA induces $\mathrm{H}_{2} \mathrm{O}_{2}$ production, thereby regulating calcium influx into guard cells, which in turn regulates changes in stomata by altering osmotic conditions [5,6,15]. In this study, we demonstrate that ATHK1 also regulates the activity of $\mathrm{Ca}^{2+}$ channels, and the NADPHase inhibitor, DPI was capable of reversing this function (Figure 3 ). These data suggest that $\mathrm{H}_{2} \mathrm{O}_{2}$-regulated channels may be controlled by ATHK1 in wild-type plants, whereas this function is lost in mutant plants.

We also performed confocal experiments to clarify the relationship between $\mathrm{ABA}, \mathrm{H}_{2} \mathrm{O}_{2}$ and $\mathrm{Ca}^{2+}$. We employed two biochemical probes, $\mathrm{H}_{2}$ DCFDA and FURA3-AM, to measure intracellular reactive oxygen species and $\mathrm{Ca}^{2+} \mathrm{lev}-$ els respectively. We found that $\mathrm{H}_{2}$ DCFDA fluorescence continuously increased in both wild-type plant and mutant guard cells in the presence of $\mathrm{ABA}$, and treatment of plant tissue with DPI decreased the trend of $\mathrm{H}_{2} \mathrm{O}_{2}$ production (Figure 2). In contrast, FURA3-AM fluorescence increased in wild-type plant guard cells but changed minimally in ATHK1 mutants, implying that ATHK1 may be an upstream regulator for $\mathrm{Ca}^{2+}$ signal transduction (Figure 4).

In addition, we also observed that ATHK1 regulated not only intracellular guard cell $\mathrm{Ca}^{2+}$ concentration, but also the rhythm of concentration variation. Interestingly, $\mathrm{Ca}^{2+}$ oscillation was only observed at high $\mathrm{Ca}^{2+}$ concentrations, such as that present in wild-type guard cells, but not at low concentrations present in ATHK1 mutant guard cells (Figure 5). This indicates that there must be a threshold of $\mathrm{Ca}^{2+}$ concentration for $\mathrm{Ca}^{2+}$ oscillation to occur, and ATHK1 may play a key role in controlling this threshold.

\section{Conclusion}

On the basis of the results presented here as well as those reported previously, we present a model describing the inter-relationships between ATHK1, ABA, $\mathrm{H}_{2} \mathrm{O}_{2}$ and $\mathrm{Ca}^{2+}$ channel signal transduction pathways. We demonstrate that the function of ATHK1 in ABA signal transduction is closely related to the activity of NADPHase, which controls 

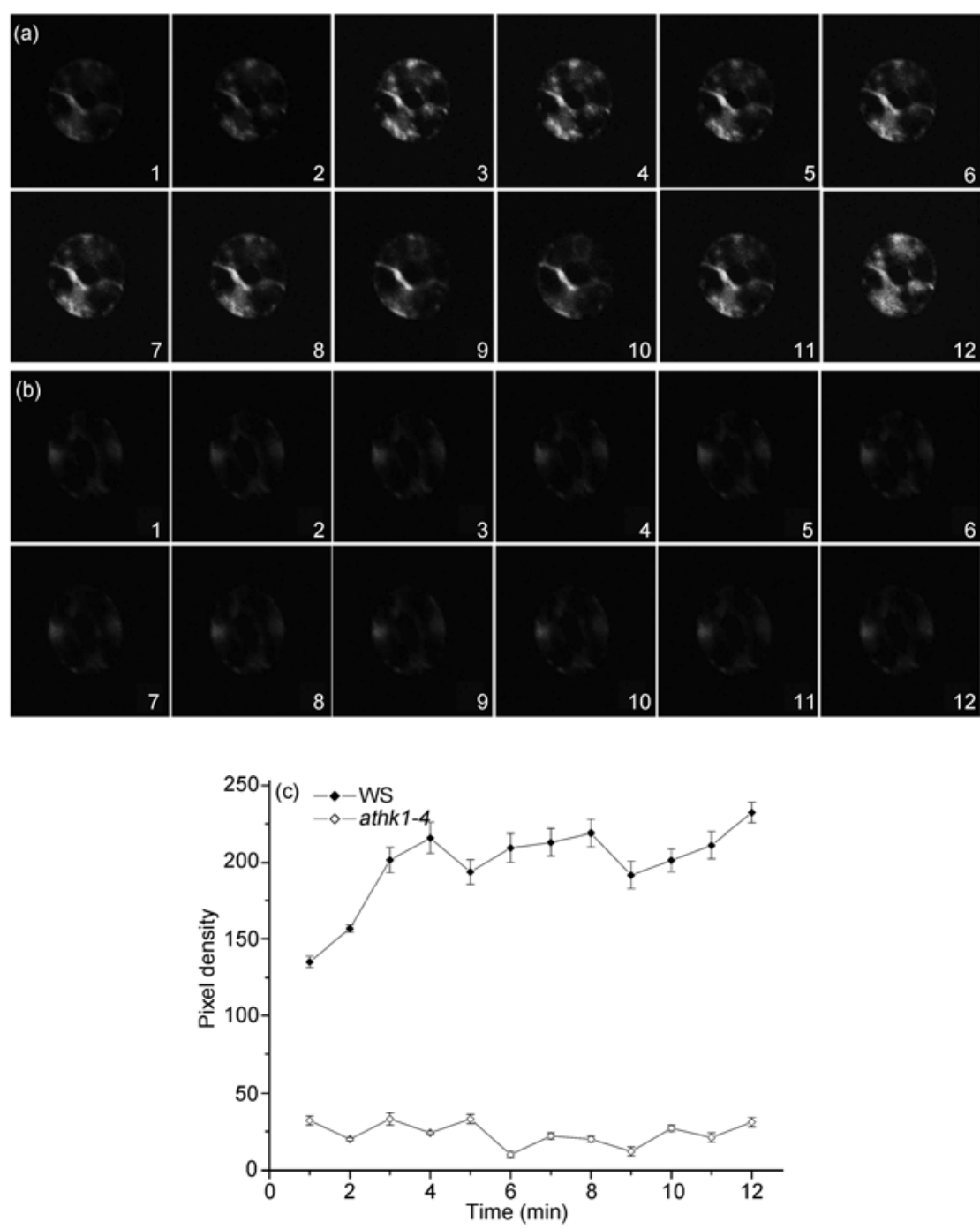

Figure 5 FURA3-AM fluorescence revealing the fluctuation of $\mathrm{Ca}^{2+}$ concentration after ABA treatment in wild-type WS and mutant ATHK1 guard cells. (a) LSCM images displaying the fluorescence levels in a WS guard cell. (b) Fluorescence levels in an ATHK1 mutant guard cell. (c) Oscillations of $\mathrm{Ca}^{2+}$ concentration as assessed by changes in FURA3-AM fluorescence pixel density in both wild-type and ATHK1 mutant plants. Data points are expressed as the mean $\pm \operatorname{SEM}(n=5, P \leqslant 0.05)$. ABA was used at a final concentration of $10 \mu \mathrm{mol} \mathrm{L}^{-1}$. Images were captured $1-12$ min after ABA treatment using a FV1000S (Olympus, Japan).

the production of $\mathrm{H}_{2} \mathrm{O}_{2}$ and directly influences $\mathrm{Ca}^{2+}$ channel activity. Thus, ATHK1 may act as a sensor for $\mathrm{H}_{2} \mathrm{O}_{2}$, transducing this signal to downstream components. ATHK1 also controlled $\mathrm{Ca}^{2+}$ channel signaling, by regulating the concentration of $\mathrm{Ca}^{2+}$ in guard cells, thus regulating stomatal closure.

This work was supported by the National Basic Research Program of China (2012CB114301) and the National Natural Science Foundation of China (31070238 and 31170252).

1 Schroeder J I, Allen G J, Hugouvieux V, et al. Guard cell signal transduction. Annul Rev Plant Physiol Plant Mol Biol, 2001, 52: 627-658

2 Wang P T, Song C P. Guard-cell signaling for hydrogen peroxide and abscisic acid. New Phytol, 2008, 178: 703-718

3 Kovtun Y, Chiu W L, Sheen J, et al. Functional analysis of oxidative stress-activated mitogen-activated protein kinase cascade in plants. Proc Natl Acad Sci USA, 2000, 97: 2940-2945

4 Pei Z M, Murata Y, Benning G, et al. Calcium channels activated by hydrogen peroxide mediate abscisic acid signaling in guard cells. Nature, 2000, 406: 731-734

5 Zhang X, Miao Y C, An G Y, et al. $\mathrm{K}^{+}$channels inhibited by hydrogen peroxide mediate abscisic acid signaling in Vicia guard cells. Cell Res, 2001, 11: 195-202

6 Zhang X, Zhang L, Dong F C, et al. Hydrogen peroxide is involved in abscisic acid-induced stomatal closure in Vicia faba. Plant Physiol, 2001, 126: 1438-1448

7 Murata Y, Pei Z M, Schroeder J I, et al. Abscisic acid activation of plasma membrane $\mathrm{Ca}^{2+}$ channels in guard cells requires cytosolic $\mathrm{NAD}(\mathrm{P}) \mathrm{H}$ and is differentially disrupted upstream and down-stream of reactive oxygen species production in abil-1 and abi2-1 protein phosphatase 2C mutants. Plant Cell, 2001, 13: 2513-2523

8 Urao T, Miyataa S, Shinozakia K Y, et al. Possible His to Asp phosphorelay signaling in an Arabidopsis two-component system. FEBS Lett, 2000, 478: 227-232 
9 Hwang I, Chen H C, Sheen J. Two-component signal transduction pathways in Arabidopsis. Plant Physiol, 2002, 129: 500-515

10 Hao G P, Wu Z Y, Chen M S, et al. ATHK1 gene regulates signal transduction of osmotic stress in Arabidopsis thaliana (in Chinese). J Plant Physiol Mol Biol, 2004, 30: 553-560

11 Pareek A, Singh A, Kumar M, et al. Whole-genome analysis of Oryza sativa reveals similar architecture of two-component signaling machinery with Arabidopsis. Plant Physiol, 2006, 142: 380-397

12 Urao T, Yakubov B, Satoh R, et al. A transmembrane hybrid-type histidine kinase in Arabidopsis functions as an osmosensor. Plant Cell, 1999, 11: 1743-1754
13 Tran L P, Urao T, Qin F, et al. Functional analysis of AHK1/ATHK1 and cytokinin receptor histidine kinases in response to abscisic acid, drought, and salt stress in Arabidopsis. Proc Natl Acad Sci USA, 2007, 104: 20623-20628

14 Wohlbach D J, Quirino B F, Sussmand M R. Analysis of the Arabidopsis histidine kinase ATHK1 reveals a connection between vegetative osmotic stress sensing and seed maturation. Plant Cell, 2008, 20: 1101-1117

15 Miao Y C, Lv D, Wang P C, et al. An Arabidopsis glutathione peroxidase functions as both a redox transducer and a scavenger in abscisic acid and drought stress responses. Plant Cell, 2006, 18: 2749-2766

Open Access This article is distributed under the terms of the Creative Commons Attribution License which permits any use, distribution, and reproduction in any medium, provided the original author(s) and source are credited. 